\title{
Functional Monomers and Polymers. LXXXIII. Synthesis of Optically Active Polymers from trans- and cis-2-Methyl-1,3-pentadienes by Asymmetric Inclusion Polymerization in Deoxycholic Acid Canals*
}

\author{
Mikiji MiYata, Yasuhiro KitAhara, and Kiichi TAKemoto \\ Department of Petroleum Chemistry, Faculty of Engineering, Osaka University, \\ Yamadakami, Suita, Osaka 565, Japan.
}

(Received April 23, 1980)

\begin{abstract}
Optically active polymers of trans- and cis-2-methyl-1,3-pentadienes were prepared by asymmetric inclusion polymerization in deoxycholic acid canals. All of the polymers prepared were found to be of 1,4-trans, head-to-tail structures and showed high optical rotations of (+) sign. It was also found that the optical activity changes reversibly with temperature.

KEY WORDS Optically Active Polymer / 2-Methyl-1,3-pentadiene / Deoxycholic Acid / Inclusion Polymerization / $\gamma$-Ray / Canal Complex /
\end{abstract}

Inclusion polymerization through the use of a chiral host molecule is a unique method for obtaining optically active polymers. ${ }^{1}$ In the preceding papers, ${ }^{2,3}$ we reported that a naturally-occurring steroidal acid, that is, deoxycholic acid $(3 \alpha, 12 \alpha-$ dihydroxy-5 $\beta$-cholan-24-oic acid) (DCA) can serve as an effective host molecule for the inclusion polymerization of substituted butadienes, particularly for the asymmetric inclusion polymerization of a prochiral monomer, trans-2-methyl-1,3pentadiene. ${ }^{4}$ In these studies, we used the conventional method for preparing DCA-canal complexes, obtained by recrystallizing DCA from methanol or ethanol containing the guest molecules. This method, however, seems to have a drawback in that the inclusion polymerization could not give optically active polymers in a high yield in the case of trans-2-methyl-1,3-pentadiene.

In the course of successive investigation, ${ }^{5}$ we found that DCA-canal complexes can be formed in a more convenient, modified way by incorporating the guest molecules into a DCA-canal without using solvents, resulting in a substantially high yield. It was further found that this new technique can be applied to the inclusion polymerization of other

* For Part LXXXII. of this series, see ref 12. prochiral monomers.

The present paper deals with the preparation of DCA-canal complexes of both trans- and cis-2methyl-1,3-pentadienes and the asymmetric inclusion polymerization of the complexes by $\gamma$-ray irradiation. The changes in the optical activity of the polymers with temperature are also considered.

\section{EXPERIMENTAL}

trans-2-Methyl-1,3-pentadiene was prepared by the pyrolysis of 2-methyl-1,3-pentadiene cyclic sulfone, which was obtained starting from 2-methyl1,3-pentadiene, according to the literature. ${ }^{6,7}$ cis-2Methyl-1,3-pentadiene was prepared by the photosensitized isomerization of trans-2-methyl-1,3pentadiene followed by the separation of the resulting cis monomer by preparative gas chromatography using $\beta, \beta^{\prime}$-oxydipropionitrile as the liquid phase. $^{8}$ These monomers were confirmed to be of more than $99 \%$ purity by gas chromatography. Commercially available DCA was purified by recrystallization from acetone to give the DCAacetone canal complex. Solvent-free DCA was then obtained by heating in vacuum at $100^{\circ} \mathrm{C}$ for $16 \mathrm{~h}(3$ Torr).

The DCA-monomer canal complexes were pre- 
pared as follows: $1.0 \mathrm{~g}$ of DCA and $0.50 \mathrm{~cm}^{3}$ of diene monomer were placed in a glass tube $(10 \mathrm{~mm}$ diameter), which was then sealed in vacuum $\left(10^{-3}\right.$ Torr) after three freeze-thaw cycles. The sealed tubes were allowed to stand for more than $20 \mathrm{~h}$ at $25^{\circ} \mathrm{C}$, in order to form the DCA-monomer canal complexes.

Irradiation was provided by $\gamma$-ray from a ${ }^{60} \mathrm{Co}$ source at $0^{\circ} \mathrm{C}$ for $2 \mathrm{~h}$ with a total dose of $1.0 \mathrm{Mrad}$. After the irradiation, the tubes were warmed to $50^{\circ} \mathrm{C}$ for 2 days in order to allow the contents to postpolymerize. The contents were then poured into excess methanol to separate the DCA from the polymers formed. The polymers thus obtained were washed thoroughly with hot methanol and dried under reduced pressure.

\section{RESULTS AND DISCUSSION}

The asymmetric inclusion polymerization of DCA-canal complexes of trans- and cis-2-methyl1,3-pentadienes was studied in a way similar to the case of the canal complex of 2,3-dimethyl-1,3butadiene. ${ }^{5}$ The DCA-monomer canal complexes were at first prepared by incorporating the monomer molecules into vacant canals of solvent-free DCA crystals, and then the monomers present in the canals were postpolymerized by heating after $\gamma$-ray irradiation. During the procedure, the crystalline DCA-canal complexes kept their original shape and appearance. The melting points of the DCApolymer canal complexes obtained after postpolymerization, $175-178^{\circ} \mathrm{C}$, were almost the same as that of DCA itself $\left(176-178^{\circ} \mathrm{C}\right)$, but different from the polymerization of other diene monomers, such as 2,3-dimethyl-1,3-butadiene $\left(213-215^{\circ} \mathrm{C}\right)$ and 1,3 -pentadiene $\left(193-195^{\circ} \mathrm{C}\right) .^{3}$

The methanol-insoluble solid obtained after the extraction of DCA with boiling methanol was found to consist exclusively of stereoregular and optically active polymers. It was also found that no polymerization proceeded outside of the DCA canals; that is, the polymer yield was less than $1.0 \mathrm{mg}$ per $0.50 \mathrm{~cm}^{3}$ of monomer in the absence of DCA under the conditions studied. The polymer yield inside the canals attained $80-100 \mathrm{mg}$ per $1.0 \mathrm{~g}$ of DCA. This corresponds to about five times more than that obtained by the earlier procedure. ${ }^{4}$

Table I summarizes the properties of the polymers thus obtained. The polymer from the $c i s$ isomer showed a higher melting point and less solubility than that from the trans isomer; the latter was soluble in carbon tetrachloride and chloroform, while the former was insoluble in chloroform. It was also found that the polymers were all of 1,4-trans, head-to-tail structure, which was confirmed by infrared (IR), ${ }^{1} \mathrm{H}$, and ${ }^{13} \mathrm{C}$ nuclear magnetic resonance (NMR) spectroscopies. The IR spectra of the polymers were almost identical with that of the 1,4-trans tactic polymer reported by Cuzin et al. ${ }^{7}$ In the ${ }^{1} \mathrm{H}$ NMR spectra, the olefinic proton signal appeared at $\delta=5.11 \mathrm{ppm}$ (in $o$-dichlorobenzene) and was assigned to the 1,4-structure. In the ${ }^{13} \mathrm{C}$ NMR spectra, only six peaks, assigned to the headto-tail, 1,4-trans structure, were observed at $\delta=16$, $20,31,48,132$, and 133 ppm (in chloroform-d).

As can be seen from Table I, the polymers show high optical rotations of the same sign as that of

Table I. Properties of the polymers obtained by asymmetric inclusion polymerization in DCA-canal complexes

\begin{tabular}{|c|c|c|c|c|c|}
\hline \multirow{2}{*}{ Monomer $^{\mathrm{a}}$} & \multirow{2}{*}{$\begin{array}{c}\mathrm{MW}^{\mathrm{b}} \\
\left(\times 10^{-4}\right)\end{array}$} & \multicolumn{3}{|c|}{ Microstructure $/ \% \mathrm{c}$} & \multirow{2}{*}[\alpha]{$_{\mathrm{D}}^{20 \mathrm{~d}}$} \\
\hline & & 1,4-trans & $1,4-c i s$ & 1,2 & \\
\hline trans-MPD & 1.4 & $>99$ & 0 & 0 & $+90^{\circ}$ \\
\hline $\begin{array}{l}\text { Mixture of trans- } \\
\text { and cis-MPD }(1: 1)\end{array}$ & 1.9 & $>99$ & 0 & 0 & $+160^{\circ}$ \\
\hline$c i s-\mathrm{MPD}$ & 3.8 & $>99$ & 0 & 0 & $+320^{\circ}$ \\
\hline
\end{tabular}

a MPD, 2-methyl-1,3-pentadiene.

b Molecular weight measured by vapor-pressure osmometry.

c Measured by ${ }^{1} \mathrm{H}$ and ${ }^{13} \mathrm{C}$ NMR spectroscopies.

d In carbon tetrachloride solution. 
DCA itself $\left([\alpha]_{D}=+55^{\circ}\right.$ in ethanol). The polymer from the cis isomer showed about three times the specific rotation value of the trans isomer. The values are about one order higher than those of poly(1,3-pentadiene). Poly(cis-1,3-pentadiene) obtained by the inclusion polymerization in DCA canals showed a specific rotation of $-21^{\circ}\left([\alpha]_{D}\right.$, in chloroform), ${ }^{9}$ and poly(trans-1,3-pentadiene) obtained by the inclusion polymerization in chiral perhydrotriphenylene showed a value of $+7.5^{\circ}$ $\left([\alpha]_{D}\right.$, in chloroform) ${ }^{10}$ With the aid of ozonolysis of the resulting poly(cis-2-methyl-1,3-pentadiene) followed by treatment with 2,4-dinitrophenylhydrazine, it was found that the optical yield was about $20 \%{ }^{11}$

Figure 1 shows the temperature dependence of the specific rotation values of these polymers. As can be seen from the figure, the optical activity tends to decrease gradually with rise in temperature, and disappears at about $60^{\circ} \mathrm{C}$. On cooling the solution again, however, the values revert to the original ones. Furthermore, the reduced viscosities $\left(\eta_{\mathrm{sp}} / c\right)$ of the resulting polymers in carbon tetrachloride tend to decrease gradually with a rise in temperature, and the values revert to the original ones on cooling again. Thus, it became evident that the optical activity and viscosity change reversibly with temperature. These results suggest the reversible conformational change of the polymer chains in solution.

A detailed study is now in progress, and the results will be reported in the near furture.

Acknowledgements. The authors wish to thank the Radiation Laboratory, the Institute of the Scientific and Industrial Research, Osaka University, for allowing them to use the ${ }^{60} \mathrm{Co}$ facilities.

\section{REFERENCES}

1. K. Takemoto and M. Miyata, J. Macromol. Sci., Rev. Maromol. Chem., C18, 86 (1980).

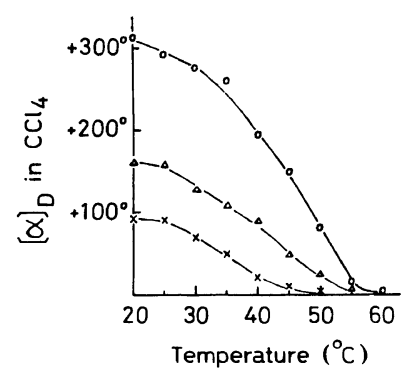

Figure 1. Temperature dependence of the specific rotation of poly(2-methyl-1,3-pentadiene) obtained by inclusion polymerization in DCA-canal complexes. Monomers: $(\times)$ trans-2-methyl-1,3-pentadiene, $(\bigcirc)$ cis2-methyl-1,3-pentadiene, and $(\triangle)$ mixture of cis- and trans-2-methyl-1,3-pentadienes $(1: 1)$.

2. M. Miyata and K. Takemoto, J. Polym. Sci., Polym. Lett. Ed., 13, 221 (1975).

3. M. Miyata and K. Takemoto, J. Polym. Sci., Polym. Symp., 55, 279 (1976).

4. M. Miyata and K. Takemoto, Polym. J., 9, 111 (1977).

5. M. Miyata and K. Takemoto, Makromol. Chem., 179, 1167 (1978).

6. G. B. Bachman and C. G. Goebel, J. Am. Chem. Soc., 64, 787 (1942).

7. D. Cuzin, Y. Chauvin, and G. Lefebvre, Eur. Polym. J., 3, 581 (1967); ibid., 5, 283 (1969).

8. H. M. Frey and R. J. Ellis, J. Chem. Soc., 4770 (1965).

9. G. Audisio and A. Silvani, J. Chem. Soc., Chem. Commun., 481 (1976).

10. M. Farina, "Inclusion Polymerization," in "Proceedings of the International Symposium on Macromolecules (IUPAC), Rio de Janeiro, 1974," E. B. Mano, Ed., Elsevier Publishers, Amsterdam, 1975, pp 21-39.

11. M. Miyata, A. Wada, and K. Takemoto, unpublished data.

12. M. Miyata, S. Senda, and K. Takemoto, Technol. Rep. Osaka Univ., 30, 581 (1980). 